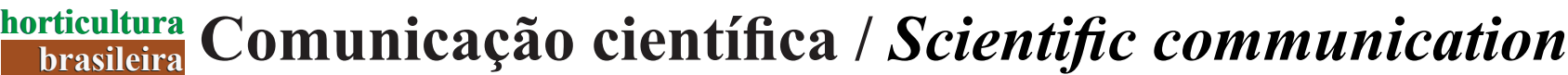

CARPENEDO, S; ANTUNES, LEC; TREPTOW, RO. 2016. Caracterização sensorial de morangos cultivados na região de Pelotas. Horticultura Brasileira 34: 565-570. DOI - http://dx.doi.org/10.1590/S0102-053620160417

\section{Caracterização sensorial de morangos cultivados na região de Pelotas}

\author{
Silvia Carpenedo'; Luis EC Antunes²; Rosa O Treptow ${ }^{3}$
}

${ }^{1}$ Universidade Federal de Pelotas (UFPel), Pelotas-RS, Brasil; carpenedo.s@hotmail.com; ${ }^{2}$ Embrapa Clima Temperado, Pelotas-RS, Brasil; luis.antunes@embrapa.br; ${ }^{3}$ Autônoma, Pelotas-RS, Brasil; rotreptow@hotmail.com

\section{RESUMO}

No Brasil as cultivares de morangueiro utilizadas, são oriundas de programas de melhoramento genético de outros países, e apresentam características sensoriais que atendem à demanda do local de origem. Além disto, essas características podem ser variáveis com as condições de clima e solo, bem como, com o sistema de cultivo adotado, sendo de grande importância a avaliação desses materiais no local de cultivo. O objetivo do trabalho foi caracterizar sensorialmente morangos das cultivares Camarosa, Diamante, Aromas, Ventana, Albion, Camino Real, Earlibrite e Festival produzidas em Pelotas-RS. Para tanto, os frutos foram avaliados quanto à aparência, textura e sabor através do Método Descritivo Quantitativo, por uma equipe composta de oito provadores treinados. A cultivar Camino Real mostrou melhor coloração enquanto a 'Earlibrite' se caracterizou pela maior intensidade de brilho e a 'Camarosa' exibiu maior quantidade de defeitos, o que resultou em comprometimento da qualidade visual. A cultivar Albion, seguida da Ventana e da Camino Real apresentou frutos de qualidade superior às demais, além de ter mostrado boas características sensoriais ao serem produzidas na região de Pelotas.

Palavras-chave: Fragaria x ananassa, morangueiro, qualidade, cultivares.

\begin{abstract}
Sensory characterization of strawberry grown in the region of Pelotas (Brazil)

The strawberry cultivars used in Brazil are from breeding programs of other countries, and have sensory characteristics that meet the demand of the place of origin. Furthermore, these characteristics may vary with the climate and soil conditions, as well as with the cropping system, for these reasons is very important to evaluate the materials in place of cultivation. The objective was to perform the sensory characterization of the strawberry cultivars Camarosa, Diamante, Aromas, Ventana, Albion, Camino Real, Earlibrite and Festival produced in Pelotas, Brazil. So, the fruits were evaluated for appearance, texture and flavor through the quantitative descriptive analysis, by a team of eight trained panelists. Camino Real cultivar showed the best color while 'Earlibrite' was characterized by the highest brightness intensity whereas Camarosa exhibited the greatest number of defects, resulting in impairment of visual quality. The cultivar Albion, followed by Ventana and Camino Real presented fruits with superior quality than the others, and they showed good sensory characteristics when produced in Pelotas region.
\end{abstract}

Keywords: Fragaria x ananassa, strawberry, quality, cultivars.

(Recebido para publicação em 12 de junho de 2015; aceito em 1 de abril de 2016) (Received on June 12, 2015; accepted on April 1, 2016)

$\mathrm{O}$ morangueiro (Fragaria $x$ ananassa) é bastante cultivado no Brasil. No entanto, todas as cultivares utilizadas para a produção de frutas são oriundas principalmente de programas de melhoramento dos Estados Unidos; logo, não existem cultivares desenvolvidas especialmente para as condições de clima e solo brasileiros.

$O$ fato destes materiais serem cultivados em um local para o qual eles não foram desenvolvidos faz com que seja necessário avaliá-los, não só quanto à adaptabilidade às condições edafoclimáticas e sistema de manejo, mas também quanto à qualidade da fruta para consu- mo local, pois, além do comportamento produtivo, a qualidade sensorial também pode ser afetada devido a diversos fatores como as interações que ocorrem entre o genótipo e o ambiente (Whitaker, 2011), sistema de cultivo (Camargo et al., 2011), ponto de maturação (Crisosto et al., 2010) e época de colheita (Hasing et al., 2013).

Estes estudos são importantes por fornecerem aos produtores e consumidores mais informações sobre a qualidade das frutas, visto que nos últimos anos, a preocupação dos consumidores em relação à qualidade dos alimentos tem crescido bastante.
Qualidade de alimento é um conceito complexo e normalmente medido utilizando-se índices objetivos relacionados a características nutricionais, microbiológicas ou físico-químicas, no entanto, quando a qualidade é definida por grau de excelência, nenhum destes índices é adequado (Cardello, 1995), pois qualidade de um alimento é considerada pela maioria dos consumidores como os atributos percebidos pelos sentidos.

Entender e identificar os fatores de qualidade percebida pelo consumidor é fundamental quando se pretende não apenas produzir alimentos, mas também comercializá-los (Trevisan et al., 2010), 
e essa identificação pode ser realizada através de análise sensorial (Cuquel et al., 2012).

A qualidade de morangos não é conceituada somente pela uniformidade, tamanho e coloração das frutas, mas também é o resultado de um complexo balanço entre doçura, aroma, textura (Jouquand et al., 2008) e valor nutricional (Resende et al., 2008), porém, sua aquisição é feita principalmente a partir de características de aparência como cor, forma, peso e do frescor do produto (Lunati, 2006).

Em razão disso, objetivou-se com o presente trabalho realizar a caracterização sensorial de morangos de diferentes cultivares introduzidas no Brasil, produzidas nas condições climáticas de Pelotas.

\section{MATERIAL E MÉTODOS}

O trabalho foi desenvolvido no mês de novembro do ano de 2009, no Labo- ratório de Ciência, Tecnologia e Análise Sensorial de Alimentos da Embrapa Clima Temperado, Pelotas-RS.

Os frutos foram cultivados sob o sistema convencional no solo, em canteiros cobertos por filme plástico preto com 0,04 $\mathrm{mm}$ de espessura. Sobre os canteiros, foram instalados túneis baixos, com $80 \mathrm{~cm}$ de altura, cobertos com polietileno transparente de baixa densidade com 0,1 mm de espessura. A irrigação foi realizada através de sistema de gotejamento. Foram utilizados morangos das cultivares Camarosa, Diamante, Aromas, Ventana, Albion e Camino Real, desenvolvidas pela Universidade da Califórnia, e das cultivares Earlibrite e Festival, desenvolvidas pela Universidade da Flórida.

A seleção da equipe para avaliação sensorial da qualidade das cultivares de morango foi realizada a partir de quatorze candidatos, com experiência em avaliações sensoriais, integrantes do quadro de funcionários e estagiários da Embrapa Clima Temperado. O poder discriminativo dos candidatos foi avaliado através de testes pareados, utilizando-se frutas de morango em diferentes estádios de maturação (ABNT, 1994), cujos resultados eram aplicados à análise sequencial de Wald mediante o sistema de decisão obtido com as retas de aceitação $\left(A_{n}=H_{0}+S_{n}\right)$ e rejeição $\left(\mathrm{R}_{\mathrm{n}}=-\mathrm{H} 1+\mathrm{Sn}\right)$, calculadas a partir de parâmetros estatísticos: $\mathrm{P} 0=0,35$ (máxima habilidade aceitável), $\mathrm{P} 1=0,65$ (mínima habilidade aceitável), $\infty=0,05$ (probabilidade de aceitar um candidato sem acuidade). As equações fornecendo os valores de inclinação da reta $(\mathrm{S})$ e as variáveis independentes da reta e de aceitação $\left(\mathrm{H}_{0}\right)$ e rejeição $\left(\mathrm{H}_{1}\right)$ foram determinadas segundo Shirose \& Mori (1984). Ao final, foram selecionados oito candidatos os quais compuseram a equipe de julgadores.

O levantamento da terminologia foi realizado pelos candidatos selecionados, utilizando-se o Método de Rede Kelly (Moskowitz, 1983). Os julgadores receberam amostras aos pares e solicitou-se

Tabela 1. Atributos, definições e referências utilizados para avaliação sensorial de morangos (attributes, definitions and reference standards used in the sensory analysis of strawberry fruits). Pelotas, Embrapa Clima Temperado, 2009.

\begin{tabular}{|c|c|c|}
\hline Aparência & Definição & Referências \\
\hline \multirow{2}{*}{ Cor } & \multirow{2}{*}{$\begin{array}{l}\text { Cor vermelha na superfície } \\
\text { externa do fruto }\end{array}$} & Claro: vermelho-claro \\
\hline & & Escuro: vermelho-escuro \\
\hline \multirow{2}{*}{ Uniformidade da cor } & \multirow{2}{*}{ Ausência de manchas } & Uniforme: sem manchas \\
\hline & & Desuniforme: frutos com cor irregular (manchados) \\
\hline \multirow{2}{*}{ Brilho } & \multirow{2}{*}{$\begin{array}{l}\text { Aparência brilhante na } \\
\text { superfície externa do fruto }\end{array}$} & Ausente: morangos maduros 3 dias após a colheita (murchos) \\
\hline & & Muito: morangos maduros recém colhidos \\
\hline \multirow{2}{*}{ Defeitos } & \multirow{2}{*}{$\begin{array}{l}\text { Presença de deformidades nos } \\
\text { frutos (defeitos leves e graves) }\end{array}$} & Leves: deformações e cicatrizes \\
\hline & & Graves: machucados, amassados, podridão e frutos imaturos \\
\hline \multicolumn{3}{|l|}{ Sabor } \\
\hline \multirow{2}{*}{ Gosto doce } & \multirow{2}{*}{$\begin{array}{l}\text { Sensação percebida associada à } \\
\text { presença de sacarose }\end{array}$} & Ausente: água \\
\hline & & Muito: solução de sacarose $16 \%$ \\
\hline \multirow{2}{*}{ Gosto ácido } & \multirow{2}{*}{$\begin{array}{l}\text { Sensação percebida associada à } \\
\text { presença de ácidos }\end{array}$} & Ausente: água \\
\hline & & Muito: solução de ácido cítrico $0,20 \%$ \\
\hline Sabor característico & Característico da fruta madura & Morango maduro \\
\hline \multirow{3}{*}{ Sabor estranho } & \multirow{3}{*}{$\begin{array}{l}\text { Sensação característica do } \\
\text { produto imaturo e/ou alterado }\end{array}$} & Purê de morangos maduros levemente fermentado \\
\hline & & Purê de morangos verdes \\
\hline & & Purê de morangos maduros + solução de cafeína $(0,15)$ \\
\hline \multicolumn{3}{|l|}{ Textura } \\
\hline \multirow{2}{*}{ Maciez } & \multirow{2}{*}{$\begin{array}{l}\text { Força necessária para deformar } \\
\text { as amostras entre os molares }\end{array}$} & Ausente: bala (tipo Soft) \\
\hline & & Muito: azeitona em conserva, sem caroço \\
\hline \multirow{2}{*}{ Suculência } & \multirow{2}{*}{$\begin{array}{l}\text { Quantidade de líquido liberado } \\
\text { pela amostra ao ser mastigada }\end{array}$} & Ausente: banana (1 fatia $2 \mathrm{~cm}$ ) \\
\hline & & Muito: Laranja (1 gomo) \\
\hline
\end{tabular}


que descrevessem as similaridades e diferenças entre elas em relação aos atributos de aparência, sabor e de textura. Através de um debate aberto entre os mesmos, foram selecionados os termos que melhor descrevessem os atributos para a avaliação das frutas em estudo.

Com os termos descritores definidos, juntamente com o material de referência utilizado (Tabela 1), foram elaboradas as fichas de avaliação das amostras. Estas foram constituídas de escalas não estruturadas de nove centímetros, cujos extremos constaram os termos referentes a cada atributo. Para cor, o valor 1, referia-se à cor vermelho-claro e 9 , à cor vermelho-escuro; para uniformidade da cor, $1=$ desuniforme, $9=$ uniforme; comercialização, $1=$ rejeitado, $9=$ aceito; qualidade geral, $1=$ péssima, $9=$ ótima; sabor característico e sabor estranho, $1=$ sem, $9=$ forte, e para os termos brilho, defeitos, maciez, suculência, doçura e acidez, $1=$ ausente, $9=$ muito .

A metodologia empregada para a avaliação sensorial dos morangos foi a Análise Descritiva Quantitativa, desenvolvida por Stone et al. (1974).

As frutas de cada cultivar foram colhidas pela manhã e manualmente, quando atingiram o estádio de maturação maduro, caracterizado pela epiderme de coloração completamente vermelha. No laboratório, foi realizada a seleção dos morangos, constituindo lotes uniformes (em relação á quantidade e apresentação dos frutos) e armazenados em câmara fria a $1,5^{\circ} \mathrm{C}$ e $95 \%$ de umidade por 24 horas, até o momento do preparo das amostras para avaliação e avaliadas a temperatura ambiente.

Os julgadores avaliaram as características de aparência (coloração da epiderme, uniformidade da cor, brilho e defeitos), de sabor (doçura, acidez, sabor característico, sabor estranho) e características de textura (firmeza e suculência). Também foi realizada análise da comercialização, representada pela intenção de compra, considerando-se as características visuais e táteis e da qualidade geral, considerando-se o conjunto das características de sabor e textura.

Para a avaliação dos atributos de aparência 15 frutas inteiras de cada cultivar foram dispostas em bandejas de poliestireno expandido, codificadas com algarismos de três dígitos e apresentadas aos julgadores sobre uma mesa central do laboratório de Ciência e Tecnologia de Alimentos e Análise Sensorial, com controle de iluminação.

Por ocasião das análises gustativas, as frutas foram lavadas e tiveram seu receptáculo floral retirado. Foram servidas $50 \mathrm{~g}$ de amostra em recipientes plásticos descartáveis, devidamente codificados com algarismos de três dígitos. Para essas avaliações, os julgadores foram conduzidos a cabines individuais providas de luz vermelha, de modo a mascarar possíveis diferenças relativas à aparência das amostras.

As amostras foram servidas monadicamente. Os julgadores receberam, juntamente com as amostras, água e uma ficha de avaliação contendo escalas não estruturadas de $9 \mathrm{~cm}$, onde constaram os termos definidos durante a etapa de levantamento da terminologia. Eles eram instruídos a indicar com um traço vertical, na linha da escala, a intensidade de cada característica avaliada.

O delineamento experimental foi de blocos completos casualizados, em esquema fatorial composto por oito

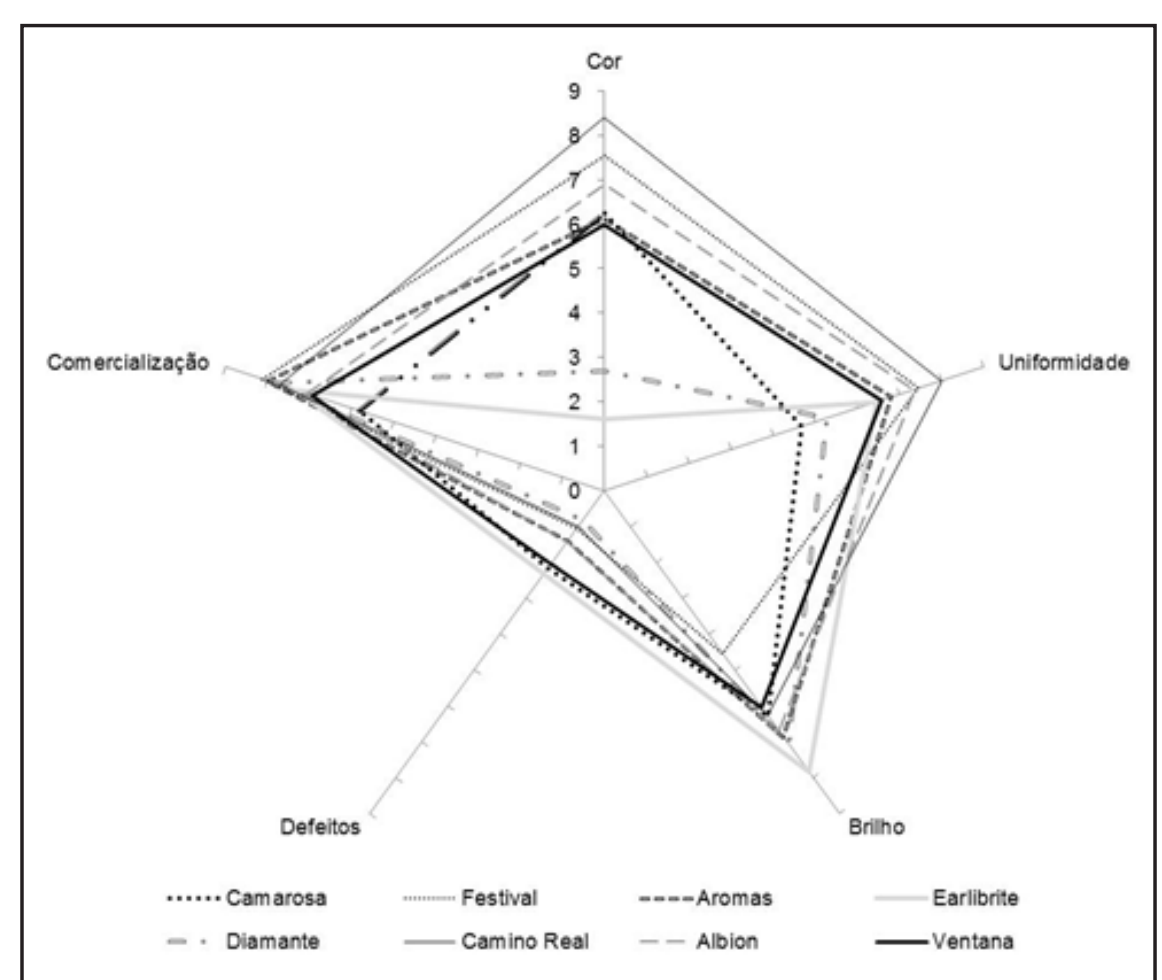

Figura 1. Perfil sensorial em gráfico radar, para os atributos de aparência em morangos de diferentes cultivares (sensory profile radar chart for appearance attributes in different strawberries cultivars). Pelotas, Embrapa Clima Temperado, 2009.

julgadores (repetições) e oito cultivares (tratamentos). Os dados foram submetidos à análise de variância e, ao teste de Tukey $(\mathrm{p} \leq 0,05)$ para a comparação de médias com o auxilio do software SISVAR (Ferreira, 2011).

\section{RESULTADOS E DISCUSSÃO}

Foram observadas diferenças na intensidade para os atributos sensoriais para as cultivares estudadas. Para o atributo cor, destacaram-se as cultivares Camino Real e Festival, as quais foram indicadas pelo maior valor na escala estruturada de $9 \mathrm{~cm}$ (Figura 1). Os morangos 'Diamante' e 'Earlibrite' receberam menores escores, indicando epiderme de coloração vermelho mais clara (Tabela 2). As cultivares Camino Real e Festival também apresentaram maior uniformidade de cor, assim como 'Albion'. A coloração vermelho mais intenso observado nos morangos 'Camino Real' e 'Festival' está de acordo com as avaliações realizadas por Vignolo et al. (2012) que encontraram teor de antocianina mais elevado destas duas cultivares 


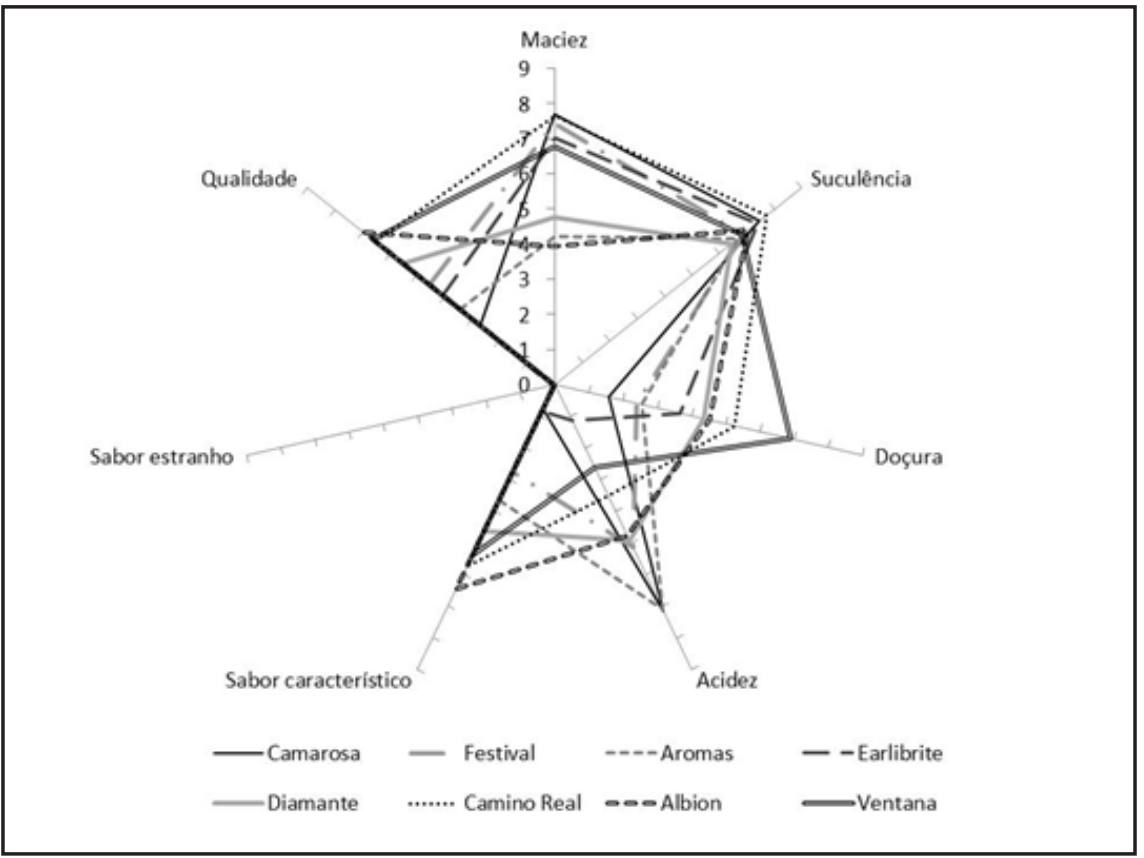

Figura 2. Perfil sensorial em gráfico radar, para os atributos de sabor e textura em morangos de diferentes cultivares (sensory profile radar chart for flavor and texture attributes of different strawberry cultivars). Pelotas, Embrapa Clima Temperado, 2009.

em relação a 'Aromas' e 'Camarosa'.

No atributo sensorial aparência, a cor é o fator de qualidade que mais atrai os consumidores de morangos (Rodas et al, 2013). Essa coloração é devida à presença de antocianinas, que são pigmentos utilizados como indicador de maturação dessa fruta para o consumo.

A cultivar Earlibrite foi a que apresentou maior intensidade de brilho, seguida por 'Diamante', 'Aromas' e 'Albion'. 'Festival' foi a que apresentou menor intensidade de brilho (Figura 1). Estes resultados estão de acordo com a descrição literária das cultivares, sendo que a cultivar Diamante (University of California, 2010), bem como Earlibrite (Chandler et al., 2000) foram descritas com coloração vermelho-brilhante tendendo ao alaranjado. $\mathrm{O}$ que indica que a diferença na coloração é uma característica inerente a cultivar.

Para a maioria das cultivares, a quantidade de frutas defeituosas variou entre 0,9 e 2,3 que é correspondente a $10 \%$ e $25 \%$, respectivamente. 'Earlibrite' e 'Camarosa' se destacaram pela maior quantidade de morangos defeituosos
$(2,3$ e 2,2$)$ enquanto 'Diamante' se caracterizou pelo menor índice.

A porcentagem de defeitos registrada nos morangos avaliados neste trabalho pode ser considerada alta, pois, levando-se em consideração a classificação dessa fruta em categorias, o lote que possui de 11 a $100 \%$ de defeitos leves, e acima de $3 \%$ até $10 \%$ de defeitos graves ficaria classificado na categoria II (CEAGESP, 2009), acarretando em um menor valor pago pelo produto por ocasião da venda.

É importante salientar que o principal defeito encontrado foi a má formação das frutas, que pode ser atribuída ao excesso de chuvas ocorrido no período da safra, o que impossibilitou a abertura regular dos túneis, operação essencial para que haja polinização das flores por agentes polinizadores, reduzindo a ocorrência deste defeito conforme constatado por Calvete et al. (2010) e Witter et al. ( 2012) nas cultivares Oso Grande, Camarosa, Diamante e Aromas.

Com relação à aceitação para comercialização, nenhuma das cultivares avaliadas foi rejeitada, porém 'Camarosa', foi a que obteve menor aceitação, com escore de 2,7 (Tabela 2). Esta menor aceitação da 'Camarosa' pode ser devido, especialmente, ao grande número de frutos defeituosos além da menor intensidade de brilho.

Em geral, os atributos de qualidade normalmente exigidos pelo consumidor para a maioria das frutas e hortaliças são sabor aroma, valor nutritivo e aparência (ausência de defeitos). Em

Tabela 2. Médias dos termos descritores para os atributos de aparência em morangos de diferentes cultivares (means of descriptors terms for appearance attributes of different strawberry cultivars). Pelotas, Embrapa Clima Temperado, 2009.

\begin{tabular}{llcccc}
\hline Cultivares & Cor & Uniformidade & Brilho & Defeitos & Comercialização \\
\hline Albion & $6,9 \mathrm{bc}$ & $7,4 \mathrm{ab}$ & $6,7 \mathrm{ab}$ & $1,4 \mathrm{abc}$ & $7,1 \mathrm{ab}$ \\
Aromas & $6,1 \mathrm{c}$ & $6,8 \mathrm{~b}$ & $6,9 \mathrm{ab}$ & $1,4 \mathrm{abc}$ & $8,0 \mathrm{a}$ \\
Camarosa & $6,2 \mathrm{c}$ & $4,7 \mathrm{c}$ & $6,3 \mathrm{~b}$ & $2,2 \mathrm{a}$ & $5,8 \mathrm{~b}$ \\
Camino Real & $8,4 \mathrm{a}$ & $8,0 \mathrm{a}$ & $6,3 \mathrm{~b}$ & $1,0 \mathrm{bc}$ & $7,7 \mathrm{a}$ \\
Diamante & $2,7 \mathrm{~d}$ & $5,3 \mathrm{c}$ & $7,1 \mathrm{ab}$ & $0,8 \mathrm{c}$ & $7,8 \mathrm{a}$ \\
Earlirite & $1,6 \mathrm{~d}$ & $6,5 \mathrm{~b}$ & $7,8 \mathrm{a}$ & $2,3 \mathrm{a}$ & $7,3 \mathrm{a}$ \\
Festival & $7,5 \mathrm{ab}$ & $7,5 \mathrm{ab}$ & $4,5 \mathrm{c}$ & $1,1 \mathrm{bc}$ & $8,1 \mathrm{a}$ \\
Ventana & $6,0 \mathrm{c}$ & $6,6 \mathrm{~b}$ & $6,0 \mathrm{~b}$ & $2,1 \mathrm{ab}$ & $7,0 \mathrm{ab}$ \\
\hline Média geral & 5,7 & 6,6 & 6,4 & 1,5 & 7,4 \\
\hline
\end{tabular}

Médias seguidas da mesma letra na coluna, não diferem entre si pelo teste de Tukey $(\mathrm{p}<0,05)$ (means in a row followed by same letters do not differ, Tukey, 5\%). 
Tabela 3. Médias dos termos descritores dos atributos de sabor e textura de morangos de diferentes cultivares (means of descriptors terms of flavor and texture attributes of strawberry cultivars). Pelotas, Embrapa Clima Temperado, 2009.

\begin{tabular}{lccccccc}
\hline Cultivares & Maciez & Suculência & Doçura & Acidez & $\begin{array}{c}\text { Sabor } \\
\text { característico }\end{array}$ & $\begin{array}{c}\text { Sabor } \\
\text { estranho }\end{array}$ & Qualidade \\
\hline Albion & $3,9 \mathrm{~b}$ & $7,0 \mathrm{abc}$ & $4,5 \mathrm{bc}$ & $4,8 \mathrm{~b}$ & $6,5 \mathrm{a}$ & $0,0^{*}$ & $6,9 \mathrm{a}$ \\
Aromas & $4,2 \mathrm{~b}$ & $6,7 \mathrm{bc}$ & $2,6 \mathrm{~d}$ & $7,1 \mathrm{a}$ & $3,7 \mathrm{~d}$ & 0,0 & $3,5 \mathrm{e} \mathrm{f}$ \\
Camarosa & $7,7 \mathrm{a}$ & $7,5 \mathrm{ab}$ & $1,6 \mathrm{~d}$ & $7,1 \mathrm{a}$ & $0,8 \mathrm{f}$ & 0,0 & $2,7 \mathrm{f}$ \\
Camino Real & $7,6 \mathrm{a}$ & $7,7 \mathrm{a}$ & $5,3 \mathrm{~b}$ & $3,5 \mathrm{c}$ & $5,7 \mathrm{ab}$ & 0,0 & $6,5 \mathrm{ab}$ \\
Diamante & $4,8 \mathrm{~b}$ & $6,4 \mathrm{c}$ & $4,3 \mathrm{bc}$ & $4,9 \mathrm{~b}$ & $4,6 \mathrm{c}$ & 0,0 & $5,6 \mathrm{bc}$ \\
Earlirite & $7,0 \mathrm{a}$ & $7,4 \mathrm{ab}$ & $3,7 \mathrm{c}$ & $1,2 \mathrm{~d}$ & $0,8 \mathrm{f}$ & 0,1 & $4,1 \mathrm{de}$ \\
Festival & $7,5 \mathrm{a}$ & $6,9 \mathrm{abc}$ & $2,4 \mathrm{~d}$ & $5,1 \mathrm{~b}$ & $2,8 \mathrm{e}$ & 0,0 & $4,6 \mathrm{~cd}$ \\
Ventana & $6,8 \mathrm{a}$ & $6,8 \mathrm{bc}$ & $6,9 \mathrm{a}$ & $2,6 \mathrm{c}$ & $5,4 \mathrm{bc}$ & 0,0 & $6,6 \mathrm{ab}$ \\
\hline Média & 6,2 & 7,1 & 3,9 & 4,6 & 3,8 & 0,0 & 5,1 \\
\hline
\end{tabular}

Médias seguidas da mesma letra na coluna, não diferem entre si pelo teste de Tukey $(\mathrm{p}<0,05)$ (means in a row followed by same letters do not differ, Tukey, 5\%).

análise sensorial de morangos, as características de aparência, principalmente os defeitos, são as mais relevantes no julgamento quando a característica avaliada é frescor da fruta (Péneau et al., 2007). Os defeitos, na maioria dos casos, são os maiores responsáveis pela aceitação visual de uma fruta. Da mesma forma que o morango, em pêssegos, a aceitação do produto pelo consumidor final, avaliada em diferentes locais, se deu preferencialmente pela ausência de defeitos (Trevisan et al., 2010).

A simulação da comercialização indicou que a presença de defeitos é determinante na aceitação ou não do morango, assim como a intensidade da coloração e o brilho, o que justifica a menor aceitação da cultivar Camarosa, uma vez que esta apresentou o maior índice de defeitos. Embora a 'Earlibrite' e a 'Camarosa' tenham apresentado escores semelhantes para defeitos, 'Earlibrite' obteve aceitação superior, o que pode ser devido à maior intensidade de brilho (Tabela 2).

Nas condições de cultivos do presente trabalho, as cultivares Albion, Diamante e Aromas, tiveram comportamento semelhante, sendo caracterizadas como as mais firmes com escores 3,9 , 4,2 e 4,8 respectivamente. Em ensaios realizados na Flórida, local de desenvolvimento da cultivar Albion, esta se caracterizou por ser tão firme quanto a 'Diamante' e mais firme que a 'Aromas' (UCDavis, 2015).

Todas as demais cultivares tenderam a ser muito macias com escores acima de 6 (tabela 3). Para suculência, a cultivar Camino Real foi superior às demais, enquanto 'Diamante' e 'Aromas' foram as menos suculentas. (Figura 2).

As cultivares, no geral, apresentaram baixos valores para a característica de doçura, 'Ventana' e 'Camino Real' foram as mais doces, enquanto a 'Camarosa' foi a que apresentou menor doçura.

Mesmo não sendo doces, não foram detectados altos níveis de acidez por parte dos julgadores na maioria das cultivares avaliadas, sendo que as duas que apresentaram maior acidez foram a 'Camarosa' e a 'Aromas'. A acidez no morango está relacionada com a cultivar, época da maturação (Jouquand et al., 2008), sofrendo também influência do sistema de produção adotado $(\mathrm{Ca}-$ margo et al., 2011). Tanto para a acidez quanto para os outros atributos avaliados, são vários os fatores que podem resultar em diferenças ou alterar as suas características, tais como a interação entre cultivar com o ambiente de cultivo (Aaby et al., 2012), o sistema de manejo e de cultivo adotado (Camargo et al., 2011), o local de plantio (Calvete et al., 2010), a época de colheita (Joquand et $a l, 2008)$ e as condições microclimáticas provocadas pela utilização do túnel (Resende et al., 2008).

Todas as cultivares avaliadas se caracterizaram por apresentarem baixos escores para sabor característico (Tabela 3). 'Albion', 'Camino Real', 'Ventana' e 'Diamante' foram as que apresenta- ram maiores escores para este atributo. As cultivares Camarosa e Earlibrite se distinguiram pela ausência de sabor característico. Apesar destas diferenças, não foi detectado sabor estranho nos morangos analisados.

As cultivares Albion, Camino Real e Ventana foram superiores às demais em qualidade geral. Aquelas que receberam menor aceitação foram 'Camarosa' e 'Aromas'. Provavelmente, a maior aceitação da 'Albion' e da 'Camino Real', foi devida principalmente ao sabor característico mais intenso das frutas destas cutivares, enquanto a aceitação da 'Ventana' se deve provavelmente à maior doçura de seus frutos.

Segundo Resende et al. (2008), o sabor característico do morango é um dos mais importantes aspectos de qualidade exigidos pelo consumidor, sendo o balanço entre a doçura e a acidez fortemente relacionado à percepção de sabor pelos julgadores. Esta afirmação está de acordo com os resultados obtidos no presente trabalho, pois a percepção do fraco sabor característico por parte dos julgadores pode também ter sido influenciado pelos baixos níveis de doçura e acidez encontrados nas frutas utilizadas. Esse fato justifica os maiores escores para as cultivares Albion, Camino Real e Ventana em relação à qualidade geral, pois foram estas que apresentaram melhores escores para este atributo.

Conclui-se que a cultivar Camino Real mostrou melhor coloração, en- 
quanto a 'Earlibrite' se caracterizou pela maior intensidade de brilho e a 'Camarosa' exibiu maior quantidade de defeitos, o que resultou no comprometimento da qualidade visual. A cultivar Albion, seguida da Ventana e da Camino Real apresentou frutos de qualidade superior às demais, além de ter mostrado boas características sensoriais ao serem produzidas na região de Pelotas.

\section{AGRADECIMENTOS}

Ao Conselho Nacional de Pesquisa e Desenvolvimento Tecnológico pelo apoio financeiro e à Embrapa Clima Temperado pela infraestrutura disponibilizada.

\section{REFERÊNCIAS}

AABY, K; MAZU, S; NES, A; SKREDE, G. 2012. Phenolic compounds in strawberry (Fragaria $\mathrm{x}$ ananassa Duch.) fruits: Composition in 27 cultivars and changes during ripening. Food Chemistry 132: 86-97. Disponível em http:/ dx.doi.org/10.1016/j.foodchem.2011.10.037. Acessado em: 01 de abril de 2014.

ASSOCIAÇÃO BRASILEIRA DE NORMAS TÉCNICAS. 1994. Teste de comparação pareada em análise sensorial dos alimentos e bebidas - Procedimento. NBR 13088, Rio de Janeiro.

CALVETE, EO; ROCHA, HC; TESSARO, F; CECCHETTI, D; NIENOW, AA; LOSS, JT. 2010. Polinização de morangueiro por Apis mellifera em ambiente protegido. Revista Brasileira de Fruticultura 32: 181-188.

CAMARGO, LKP; RESENDE, JTV; TOMINAGA, TT; KURCHAIDT, SM; CAMARGO, CK; FIGUEIREDO, AST. 2011. Postharvest quality of strawberry fruits produced in organic and conventional systems.
Horticultura Brasileira 29: 577-583.

CARDELLO, AV. 1995. Food quality: Relativity, context and consumer expectations. Food Quality and Preference 6: 163-170.

CEAGESP. 2009. Programa brasileiro para a modernização da horticultura. Normas de classificação de morango. São Paulo: CEAGESP (Documentos 33).

CHANDLER, CK; LEGARD, DE; DUNINGAN, DD; CROCKER, TE; SIMS, CA. 2000. 'Strawberry Earlibrite' Strawberry. HortScience 35: 1363-1365.

CRISOSTO, CH; BREMER, V; FERGUSON, L; CRISOSTO, GM. 2010. Evaluating quality attributes of four fresh fig (Ficus carica L.) cultivars harvested at two maturity stages. HortScience 45: 707-710.

CUQUEL, FL; OLIVEIRA, CFS; LAVORANTI, OJ. 2012. Sensory profile of eleven peach cultivars. Ciência e Tecnologia de Alimentos 32: 70-75.

FERREIRA, DF. 2011. SISVAR: a computer statistical analysis system. Ciência e Agrotecnologia 6: 1039-1042.

HASING, TN; OSORIO, LF; WHITAKER, VM. 2013. Within-season stability of strawberry soluble solids content. Journal of the American Society for Horticultural Science 138: 190197.

JOUQUAND, J; CHANDLER, C; PLOTTO, A; GOODNER, K. 2008. A sensory and chemical analysis of fresh strawberries over harvest dates and seasons reveals factor that affect eating quality. Journal of the American Society for Horticultural Science 133: 859-867.

LUNATI, F. 2006. Le fragole italiane in cerca di un posto al solo. Rivista di Frutticoltura e Ortofloricultura 68: 9-10.

MOSKOWITZ, HR. 1983. Product testing and sensory evaluation of foods: marketing and $R \& D$ approaches. Westport: Food \& Nutrition. 605p.

PÉNEAU, SP; BROCKHOFF, PB; ESCHER, F; NUESSLI, J. 2007. A comprehensive approach to evaluate the freshness of strawberries and carrots. Postharvest Biology and Technology 45: 20-29.

RESENDE, JTV; CAMARGO, LKP; ARGANDOÑA, EJS; MARCHESE, A;
CAMARGO, CK. 2008. Sensory analysis and chemical characterization of strawberry fruits. Horticultura Brasileira 26: 371-374.

RODAS, CL; SILVA, IP; COELHO, VAT; FERREIRA， DMG; SOUZA， RJ; CARVALHO, JG. 2013. Chemical properties and rates of external color of strawberry fruits grown using nitrogen and potassium fertigation. Idesia 31: 53-58.

SHIROSE, I; MORI, EEM. 1984. Aplicação da análise sequencial a seleção de provadores pelo teste angular. Coletânea ITAL 14: 39-35.

STONE, H; SIDEL, JL; OLIVER, S; WOOLSEY, A; SINGLETTON, RC.1974. Sensory evaluation by quantitative descriptive analysis. Food Technology 28: 24-34.

TREVISAN, R; PIANA, CFB; TREPTOW, RO; GONÇALVES, ED; ANTUNES, LEC. 2010. Perfil e preferências do consumidor de pêssego (Prunus persica) em diferentes regiões produtoras no Rio Grande do Sul. Revista Brasileira de Fruticultura 32: 90-100.

UCDavis. 2015. The UC Patented Strawberry Cultivars. Disponível em http://research. ucdavis.edu/industry/ia/industry/strawberry/ cultivars/\#albion. Acessado em 15 de novembro de 2015.

UNIVERSITY OF CALIFORNIA. 2010. The Diamante cultivar. Davis, p. 2, Disponível em: <http://www.innovationaccess.ucdavis. edu/strawberry/Diamante.htm>. Acessado em: 22 jun. 2010.

VIGNOLO, GK; ARAUJO, VF; ANTUNES, LEC; PICOLOTTO, L; VIZZOTTO, M; FERNANDES, A. 2012. Produção de frutos e compostos funcionais de quatro cultivares de morangueiro. Horticultura Brasileira 30: S3470-S3476.

WHITAKER, VM; HASING, VM; CHANDLER, CK. 2011. Historical trends in strawberry fruit quality revealed by a trial of university of Florida cultivars and advanced selections. Hortscience 46: 553-557.

WITTER, S; RADIN, R; LISBOA, BB; TEIXEIRA, JSG; BLOCHTEIN, B; IMPERATRIZ-FONSECA, VL. 2012. Desempenho de cultivares de morango submetidas a diferentes tipos de polinização em ambiente protegido. Pesquisa Agropecuária Brasileira 47: 58-65. 